\title{
Inverse gas chromatographic study of the surface properties of talc impregnated with different acidic and basic polymers
}

\author{
Marie-Pierre Comard ${ }^{\mathrm{a}}$, Rachel Calvet ${ }^{\mathrm{a}, *}$, John A. Dodds $^{\mathrm{a}}$, Henri Balard ${ }^{\mathrm{b}}$ \\ ${ }^{a}$ Ecole des Mines d'Albi-Carmaux, Campus Jarlard, route de Teillet, 81013 Albi, France \\ ${ }^{\mathrm{b}}$ Institut de Chimie des Surfaces et Interfaces, 15 rue Jean Starcky, 68057 Mulhouse, France
}

\begin{abstract}
A severe limitation of inverse gas chromatography at infinite dilution (IGC-ID) is that it is mainly sensitive to high-energy sites making it difficult to obtain information about the surface of heterogeneous solids, e.g. the talc. In this paper, it is shown that a progressive impregnation of the talc surface with a suitable polymer can saturate the high-energy sites making the probes access less energetic free sites, specifically the basal surfaces of talc. Here, such impregnations are carried out using a surfactant (cetyltrimethylammonium bromide (CTAB)), different molecular weights polymers (polyethylene glycol (PEG) 2000, 20000, 35000, and 100 000) and polymers with different acidic or basic character (polyethylvinylether (PEVE), polyvinyl chloride (PVC), polymethylvinylketone (PMVK), and polystyrene (PS)). One of the aims of this study is to estimate the ratio of lateral and basal surfaces.
\end{abstract}

Keywords: Inverse gas chromatography; Talc; Polymer; Surfactant

\section{Introduction}

Inverse gas chromatography (IGC) is a useful method for studying the surface properties of finely divided solids [1-3]. It consists of injecting clearly identified molecules, called probes, into a column packed with the solid to be tested. The injected probes undergo interactions with the solid surface as they are displaced through the column by a carrier gas stream. The complexity of these interactions depends on the amount of probe injected. Two IGC techniques may be distinguished: infinite dilution IGC, or finite solute concentration IGC. The present study has been carried out at infinite dilution (IGCID). In this case, very small amounts of probes are injected in such a way that the interactions between probe molecules themselves can be considered to be negligible and only interactions between an isolated probe and the solid surface are important. By IGC-ID, one can obtain the apparent dispersive component of the free surface energy $\gamma_{\mathrm{S}}^{\mathrm{d}}$ (injection of linear alkanes), the increment of energy linked to specific interactions (injection of polar probes) and the nanomorphological index $I_{\mathrm{m}}$ (injection of bulky probes). The latter gives

\footnotetext{
${ }^{*}$ Corresponding author.

E-mail address: calvet@enstimac.fr (R. Calvet).
}

information on the morphology of the solid at the molecular scale. In the work reported here, we use IGC-ID to study the surface properties of talc impregnated with increasing amounts of a surfactant (cetyltrimethylammonium bromide (CTAB)) or of different polymers (polyethylene glycol (PEG) or polyvinyl derivatives).

The structure of talc directs the choice of the molecules used for impregnation. Talc is a lamellar phyllosilicate with the chemical formula $\left(\mathrm{Mg}_{3} \mathrm{Si}_{4} \mathrm{O}_{10}(\mathrm{OH})_{2}\right.$ consisting of octahedral brucite sheets $\left[\mathrm{Mg}_{3}(\mathrm{OH})_{2}\right]_{n}^{2 n-}$ sandwiched between two tetrahedral silica sheets, $\left[\mathrm{Si}_{2} \mathrm{O}_{5}\right]_{n}^{2 n}$, giving superimposed layers which form the structure of talc. The bonds between two sheets are van der Waals interactions. The crystal presents basal nonfunctionalised hydrophobic surfaces with $\mathrm{Si}-\mathrm{O}-\mathrm{Si}$ groups, as well as lateral surfaces with $\mathrm{Si}-\mathrm{OH}$ groups and residual magnesium cations. The industrial use of talc depends on the surface properties of its lamellar structure and therefore on the ratio of basal to lateral surfaces. Different techniques can be used to measure these parameters: immersion calorimetry [4], adsorption of various gases (nitrogen, argon, water vapour, etc.) [5,6] and measurement of contact angles [7]. IGC-ID represents valuable addition to these techniques because, instead of giving a global measurement, it examines the solid surface at the scale of the injected probe. The variety of available 
probes then make this technique highly attractive, since the probe can be adapted to the nature of the solid provided that the probe can be vaporised in the carrier gas stream. Another important aspect of IGC-ID is that it is mainly sensitive to the presence of sites with high interaction energies. In the case of a heterogeneous solid such as talc, and because of the small amounts of probe injected, only the higher energy sites are visited by the probes. In the case of talc, the probes mainly visit the more energetic sites located on the lateral surfaces, i.e. acidic silanols $\mathrm{Si}-\mathrm{OH}$ and residual magnesium cations. The aim of the experiment protocol of coupling an impregnation of the solid surface by a polymer with IGC-ID is to progressively saturate these high-energy sites making the probes successively access less energetic sites which remain free. In the case of talc, it seemed appropriate to first impregnate the surface of this solid with a basic polymer, polyethylene glycol (PEG), as it is reasonable to suppose that this polymer will saturate the more energetic sites by preferential interactions with the acidic lateral surfaces. After the lateral surfaces have been covered by polymer, the probes will then access the less energetic sites on the basal surfaces. This method should then allow the determination of the respective amounts of lateral and basal surfaces. Conversely, impregnation with an acidic polymer displaying some affinity with the basic siloxane $\mathrm{Si}-\mathrm{O}-\mathrm{Si}$ groups on the basal surfaces of the talc should protect the high-energy sites on the lateral surfaces and lead to the values obtained on talc without any impregnation.

As the aim of this study is to develop a new method for estimating the ratio of lateral to basal surfaces; the evolution of $\gamma_{\mathrm{S}}^{\mathrm{d}}$ and $I_{\mathrm{m}}$ was followed as a function of the surfactant or polymer impregnation ratio. The dispersive component of the surface energy $\gamma_{\mathrm{S}}^{\mathrm{d}}$ is obtained by analysing the retention times of a series of linear alkanes injected as probes, and gives a measurement of the dispersive London forces. The nanomorphological index $I_{\mathrm{m}}$, obtained from the injection of cyclic or branched alkanes, gives information on the nanomorphology of the solid at the molecular scale. It is a measure of the surface of the solid that can be reached by the bulky probe. $I_{\mathrm{m}}$ reflects molecular scale size exclusion effects.

\section{Experimental}

\subsection{Materials}

The talc used was supplied by Luzenac Europe and comes from a French quarry. The mean particle size is 10 $\mu \mathrm{m}$, as measured by Micromeritics 5100 Sedigraph. The argon BET specific surface area is $3 \mathrm{~m}^{2} / \mathrm{g}$. The different PEGs were obtained from Fluka, the other polymers (polyvinyl chloride (PVC), polystyrene (PS), polymethylvinylketone (PMVK) and polyethylvinylether (PEVE)) from Aldrich and the CTAB from Janssen. The PEGs were purified by solubilisation in chloroform and precipitation in heptane.

\subsection{Experimental IGC conditions}

Two Hewlett Packard 6890 gas chromatographs, each fitted with two flame ionisation detectors (FID), were used with helium carrier gas. The injector and detector are heated to $150{ }^{\circ} \mathrm{C}$. All the columns were conditioned at $120{ }^{\circ} \mathrm{C}$ overnight and the analyses made at $100{ }^{\circ} \mathrm{C}$. Net retention times were calculated by subtracting the retention time of a nonretained molecule, methane, from the retention times of the probes.

\subsection{Preparation of the samples: controlled impregnation of talc}

A solution of surfactant or polymer of known concentration is prepared in dichloromethane (for PVC, the solvent was THF). A given volume of this solution is diluted with 5 $\mathrm{ml}$ of the same solvent. The mass of talc required to fill the column (around $2 \mathrm{~g}$ ) is stirred with the polymer solution. The mixture is then placed in a vacuum oven. Using such a procedure, solvent is evaporated and the polymer is deposited on the talc surface. The chromatography column is then filled with the impregnated talc. The percentage of polymer or surfactant per gram of talc can be determined from the volume added, the concentration of the initial solution and the mass of talc used for loading in the column.

\section{Results}

\subsection{Application of the method with PEG 20000}

Fig. 1 shows the changes in the dispersive component of the surface energy $\gamma_{S}^{\mathrm{d}}$ as a function of the PEG $20 \mathrm{M}$ impregnation ratio. This curve has four critical ratios [8]:

- For $\tau_{1}$ at $0.02 \%$ of PEG $20 \mathrm{M}$ is a first plateau corresponding to a $\gamma_{\mathrm{S}}^{\mathrm{d}}$ equal to $160 \mathrm{~mJ} / \mathrm{m}^{2}$. This high value of $\gamma_{\mathrm{S}}^{\mathrm{d}}$, also observed without polymer, is typical of lamellar structures $[9,10]$. It reflects the insertion of linear alkanes into slit-shaped sites located on the lateral surfaces. The ratio $\tau_{1}$ could correspond to the amount of polymer required to fill the lateral surfaces. In fact, the basic character of PEG $20 \mathrm{M}$ should lead to adsorption on the acidic $\mathrm{Si}-\mathrm{OH}$ groups on the lateral surfaces. The complexation properties towards cations, such as magnesium present on the lateral surfaces, will also first direct the PEG towards lateral surfaces.

- For $\tau_{2}$ at $0.084 \%$ of PEG $20 \mathrm{M}$ is a first decrease of $\gamma_{\mathrm{S}}^{\mathrm{d}}$. This decrease can be attributed to the filling of the wedge-shaped sites between talc crystallites and grain joints. The difference of $\tau_{2}-\tau_{1}$ could correspond to the amount of polymer required to cover the grain joints.

- For $\tau_{3}$ at $0.4 \%$ of PEG $20 \mathrm{M}$ is a second plateau corresponding to a $\gamma_{\mathrm{S}}^{\mathrm{d}}$ equal to $45 \mathrm{~mJ} / \mathrm{m}^{2}$. This latter value is that obtained by others [11] using Washburn's 

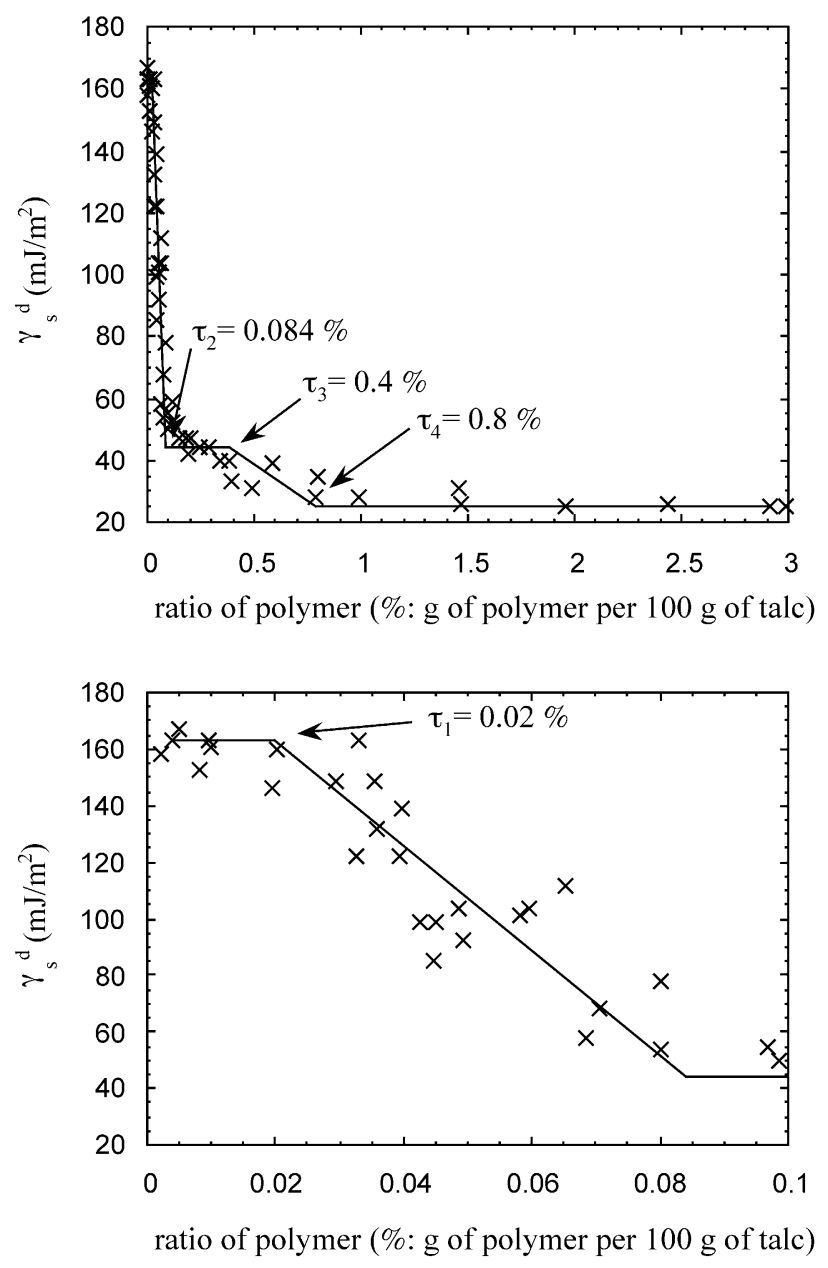

Fig. 1. Variation of the dispersive component of the surface energy $\gamma_{S}^{d}$ as a function of PEG $20 \mathrm{M}$ impregnation ratio (between $0-3 \%$ on the first graph and $0-0.1 \%$ on the second graph).

capillary rise method which, contrary to IGC-ID, is representative of the whole surface and therefore mainly sensitive to the basal surfaces that are predominant in the talc used here. From $\tau_{2}$ to $\tau_{3}$, only basal surfaces are visible to the linear alkanes. The difference of $\tau_{3}-\tau_{2}$ could then correspond to the amount of polymer required to cover basal surfaces. The basic PEG displays a low affinity for the basal surfaces formed of basic siloxane $\mathrm{Si}-\mathrm{O}-\mathrm{Si}$ bridges.

- After $\tau_{3}$, a final decrease of $\gamma_{\mathrm{S}}^{\mathrm{d}}$ down to a value of $28 \mathrm{~mJ} /$ $\mathrm{m}^{2}$ is observed for $0.8 \%$ of PEG $20 \mathrm{M}$. This value of 28 $\mathrm{mJ} / \mathrm{m}^{2}$ is characteristic of a multi-layer of PEG monomeric units entirely covering the talc surface [12]. This last decrease could correspond to the coverage of the final, less energetic, structural defects.

Fig. 2 shows the evolution of the nanomorphological index $I_{\mathrm{m}}$ as a function of the PEG $20 \mathrm{M}$ impregnation ratio. $I_{\mathrm{m}}$ increases with impregnation ratio of the polymer, thus following an opposite trend to that observed for $\gamma_{S}^{d}$. For low impregnation ratios, an $I_{\mathrm{m}}$ close to 0.1 indicates a high exclusion effect: linear alkanes can be inserted into structural defects from which cyclic alkanes are excluded due to steric constraints. As the polymer is deposited on the solid surface, this phenomenon progressively disappears as structural defects are progressively covered and $I_{\mathrm{m}}$ tends towards 1. A value higher than 1 corresponds to the absorption of the probe in a multi-layer polymer.

In the second part of this work, we study the influence of polymer molecular weight on impregnation.

\subsection{Controlled impregnation with different PEG-influence of polymer molecular weight}

The influence of the molecular weight of PEG on both $\gamma_{\mathrm{S}}^{\mathrm{d}}$ and $I_{\mathrm{m}}$ has been studied as a function of impregnation ratio for PEG 2000, 20000, 35000, and 100000. Fig. 3 displays only the variation of the $\gamma_{\mathrm{S}}^{\mathrm{d}}$ and shows that the polymer molecular weight has no influence on the evolution of the surface properties of talc as a function of

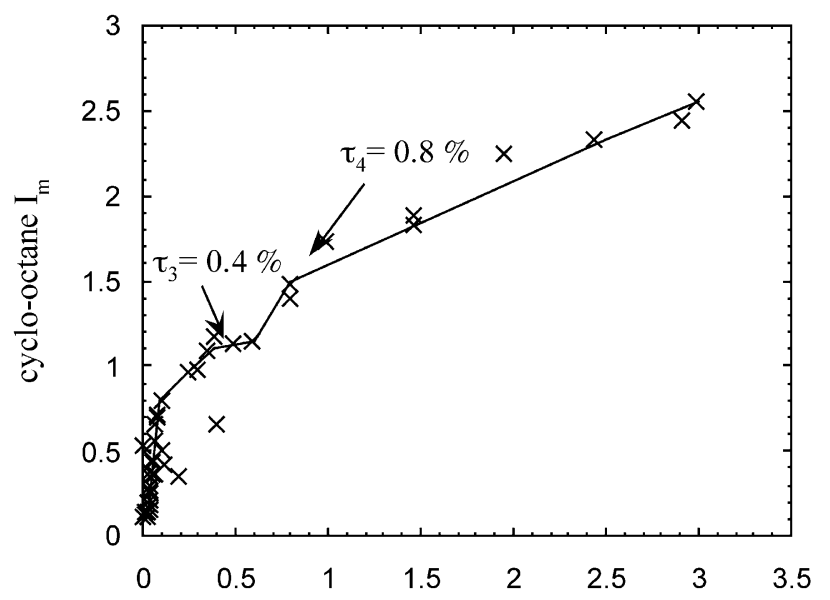

ratio of polymer (\%: $\mathrm{g}$ of polymer per $100 \mathrm{~g}$ of talc)

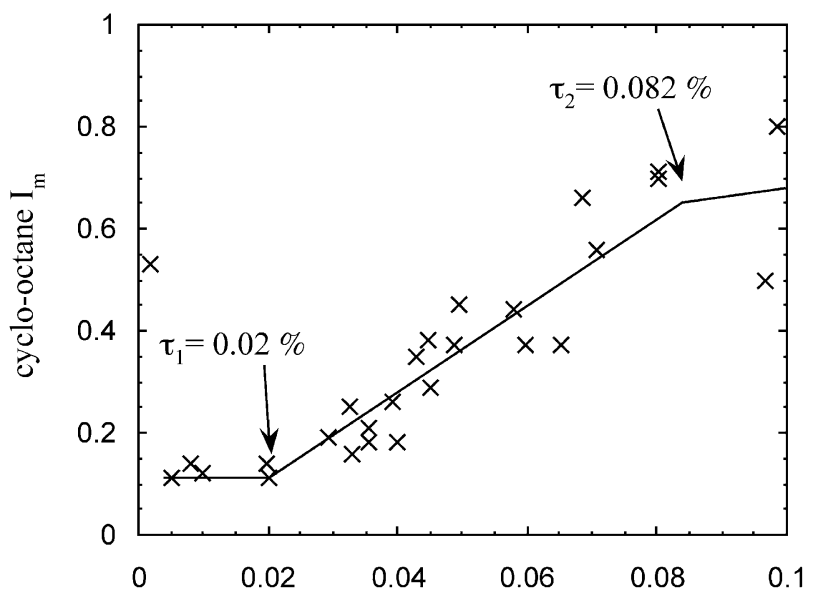

ratio of polymer (\%: $\mathrm{g}$ of polymer per $100 \mathrm{~g}$ of talc)

Fig. 2. Variation of the nanomorphological index $I_{\mathrm{m}}$ as a function of PEG $20 \mathrm{M}$ impregnation ratio (between $0-3 \%$ on the first graph and $0-0.1 \%$ on the second graph). 

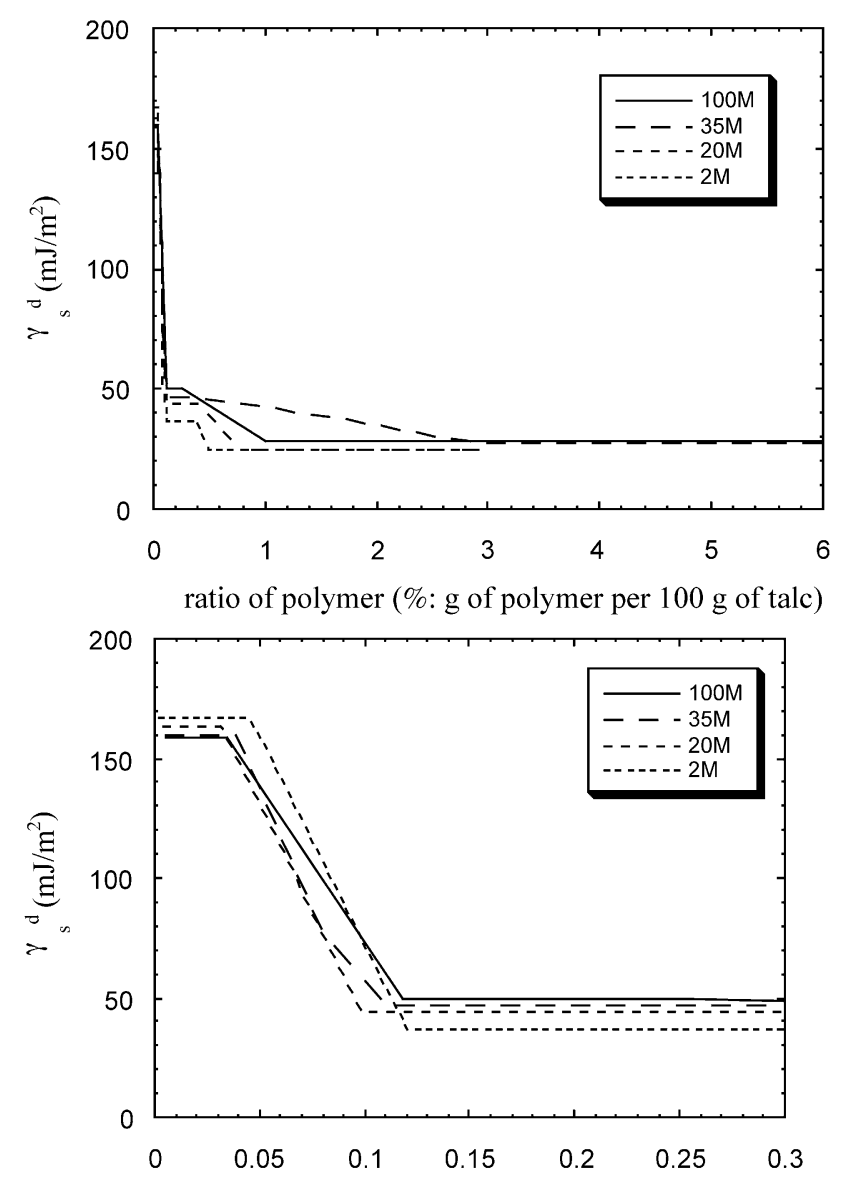

ratio of polymer (\%: g of polymer per $100 \mathrm{~g}$ of talc)

Fig. 3. Variation of the dispersive component of the surface energy $\gamma_{\mathrm{S}}^{\mathrm{d}}$ of talc impregnated with PEG 2000, 20000, 35000 and 100000 (between $0-6 \%$ on the first graph and $0-0.3 \%$ on the second graph).

impregnation ratio. A translation towards high impregnation ratios is only observed in the case of PEG 2000. This phenomenon is probably due to an overestimation of the polymer mass impregnated due to the evaporation of low molecular weight oligomers during column conditioning. This noninfluence of the molecular weight of the impregnated polymer on solid surface properties has already been observed when grafting of PEG on pyrogenic silicas [13].

\subsection{Controlled impregnation with polymers or surfactants with different acidic or basic characters}

Fig. 4 shows the changes in $\gamma_{S}^{\mathrm{d}}$ for talc impregnated with increasing ratios of basic polyethylvinyleather (PEVE), basic polystyrene (PS) and acidic cetyltrimethylammonium bromide (CTAB). The choice of this latter surfactant was guided by a previous study carried out with the same talc [14].

The nature of the impregnation agent completely changes the evolution of $\gamma_{\mathrm{S}}^{\mathrm{d}}$ as a function of impregnation ratio. Impregnation with PEVE leads to a fast decrease of $\gamma_{\mathrm{S}}{ }^{\mathrm{d}}$ down to the lowest impregnation ratio. There is no plateau for low impregnation ratios as observed with PEG. At a ratio equal to $0.19 \%, \gamma_{S}^{\mathrm{d}}$ becomes equal to $45 \mathrm{~mJ} / \mathrm{m}^{2}$ and the decrease then slows down. $\gamma_{\mathrm{S}}^{\mathrm{d}}$ continues to decrease down to $28 \mathrm{~mJ} / \mathrm{m}^{2}$ after $0.40 \%$ of PEVE. Despite the fact that PEVE is a basic polymer like PEG, these two polymers exhibit notable differences in stereochemistry (Fig. 5). PEG has a linear structure whereas PEVE displays some branchings from the linear chain and asymmetric carbons. Because of its structure, PEVE cannot fit against as rough a surface as easily as the linear PEG can.

The curve of $\gamma_{S}^{d}$ as a function of impregnation with PS presents a totally different shape compared with the two other polymers. This polymer is unable to hinder highenergy sites; the value of $\gamma_{S}^{d}$ remains steadily high up to $1-$ $1.5 \%$ of PS. The stereochemistry of the macromolecular chain and the low interaction potential with lateral surfaces can explain such behaviour. The polymer probably first covers the basal surfaces which are not visible to IGC-ID,
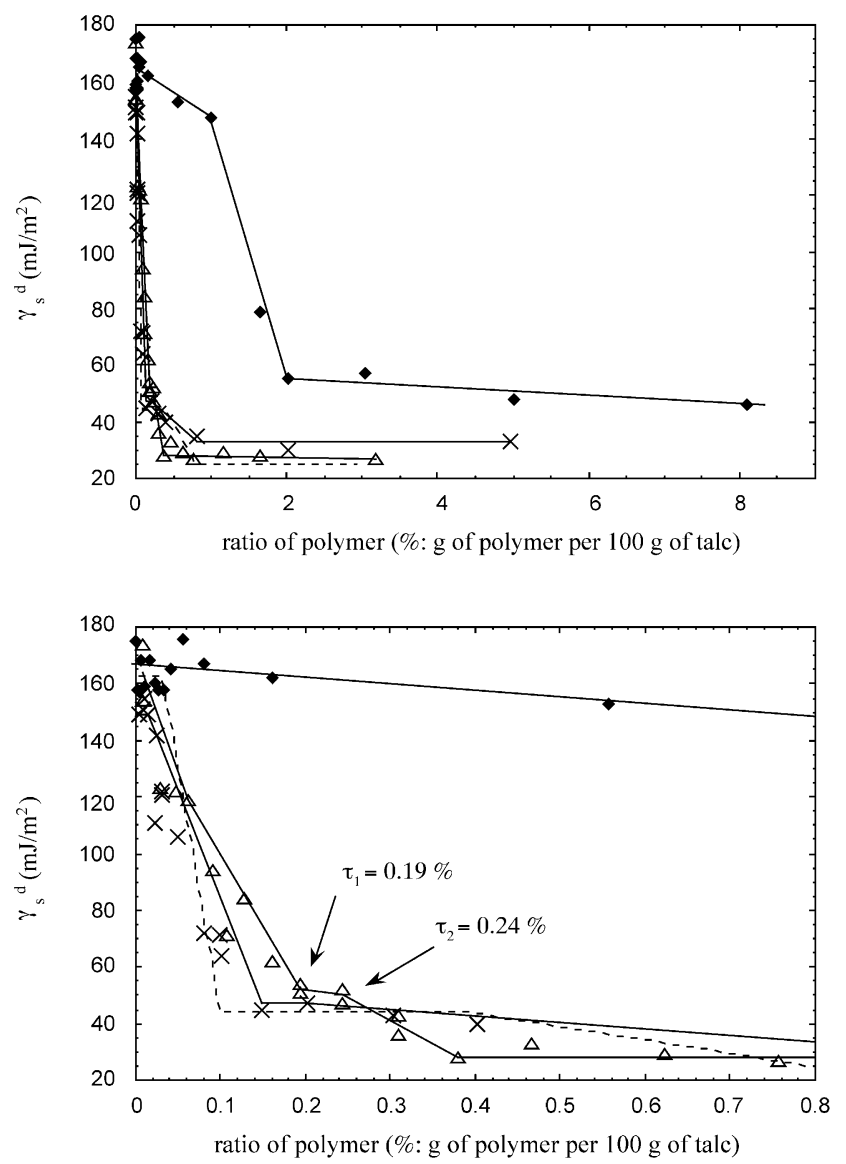

Fig. 4. Variation of the dispersive component of the surface energy $\gamma_{\mathrm{S}}^{\mathrm{d}}$ (between $0-9 \%$ on the first graph and $0-0.8 \%$ on the second graph) of talc impregnated with PEVE $(\triangle)$, PS $(\diamond)$ and CTAB $(\times)$ (the dotted curve corresponds to the $\gamma_{\mathrm{S}}^{\mathrm{d}}$ variation observed with PEG $20 \mathrm{M}$ as the reference). 


$$
-\left(\mathrm{CH}_{2}-\mathrm{CH}_{2}-\mathrm{O}\right)-
$$<smiles>CCC(C)C(Cl)CC</smiles>

PEG<smiles>CCCC(C)C(C)CC</smiles>

PMVC
PVC<smiles>CCOC1CCCCC1</smiles>

PEVE<smiles>CCCCCCCCCCCCCC</smiles>

PS

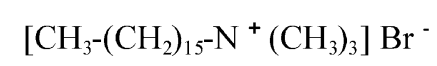

CTAB

Fig. 5. Stereochemistry of all polymers or surfactant used in the study.

rather than the lateral surfaces which alone contribute to the high value of $\gamma_{\mathrm{s}}^{\mathrm{d}}$. After $1-1.5 \%$, the amount of PS is so high that it covers almost all the surface of the microcrystallites, and $\gamma_{\mathrm{S}}^{\mathrm{d}}$ falls to reach the energy of a talc surface entirely covered with PS.

The acidic character of CTAB, due to the quaternary ammonium function, should direct its preferential adsorption onto basal surfaces, thus providing a behaviour analogous to that of PS. In fact, the insertion of the alkyl chains into the slit-shaped sites on the lateral surfaces must be responsible for the fast decrease of $\gamma_{\mathrm{S}}^{\mathrm{d}}$.

\section{Conclusion}

A new method for studying the surface of a heterogeneous solid by IGC-ID has been developed. After having saturated the high-energy sites on the solid under study by impregnation with a suitable polymer, the injected probes can visit the low-energy sites that are not visible in conventional IGC-ID. This method is first used to study the surface heterogeneity of a lamellar solid: talc. In a second stage, we obtain information on the interactions between the solid surface and different polymers or a surfactant. However, the interpretation of the results is not as simple as what might be expected from the study of the evolution of the surface properties of talc impregnated with PEG. Obviously, the nature of the polymer and its stereochemistry, or the amphiphilic character of CTAB, has a large influence on the conformation of the adsorbed macromolecules at the talc surface. Three different types of behaviour are observed.

- With PEG, four different trends were observed that can be attributed respectively to: coverage of lateral surfaces, structural defects, basal surfaces and finally, to the formation of a thick layer of PEG.
- With PEVE and CTAB, only three zones can be observed: first, a rapid decrease, followed by a slower decrease, and then, a plateau corresponding to the formation of a thick layer of macromolecules.

- With PS, the apparent surface energy of talc remains steady up to very high impregnation ratios. This behaviour can be attributed to a total lack of affinity for lateral surfaces due to both the stereochemistry and the chemistry of the polymer, in particular, the absence of hetero-elements.

Some complementary studies with different pairs of polymer/solids will be necessary to fully interpret these different behaviours.

Finally, it is important to point out that the technique described requires a polymer/solid pair that is thermally stable at the analysis temperature. This condition was not satisfied with polyvinyl chloride (PVC) and polyvinylmethylketone (PVMK) which gave erratic results that can be interpreted as being caused by the decomposition of the macromolecules catalysed by the talc surface.

\section{References}

[1] H. Balard, E. Papirer, Prog. Org. Coat. 22 (1993) 1-17.

[2] H. Balard, A. Saada, J. Hartmann, O. Aouadj, E. Papirer, Macromol. Symp. 108 (1996) 63-80.

[3] E. Papirer, H. Balard, in: A. Dabrowski, V.A. Tertykh (Eds.), Adsorption on New and Modified Inorganic Sorbents, 1996, pp. 479-502 (Chap. 2.6).

[4] L.J. Michot, F. Villiéras, M. François, J. Yvon, R. Le Dred, J.M. Cases, Langmuir 10 (1994) 3765-3773.

[5] J.M. Cases, P. Cunin, Y. Grillet, C. Poinsignon, J. Yvon, Clay Miner. 21 (1) (1986) 55-68.

[6] F. Bardot, F. Villiéras, L.J. Michot, M. François, G. Gérard, J.M. Cases, J. Dispers. Sci. Technol. 19 (6-7) (1998) 739-759.

[7] R.F. Giese, P.M. Costanzo, C.J. van Oss, Phys. Chem. Miner. 17 (1991) 611-616. 
[8] M.P. Comard, R. Calvet, S. Del-Confetto, H. Balard, J.A. Dodds, Macromol. Symp. 169 (2001) 19-34.

[9] G. Ligner, A. Vidal, H. Balard, E. Papirer, J. Colloid Interface Sci. 133 (1) (1989) 200-210

[10] A. Saada, E. Papirer, H. Balard, B. Siffert, J. Colloid Interface Sci. 175 (1995) 212-218.

[11] M.E. Schrader, S. Yariv, J. Colloid Interface Sci. 136 (1) (1990) $85-94$.
[12] H. Balard, D. Yeates, E. Papirer, M. Gastiger, P. Bouard, F. Clauss, R. Baeza, MOFFIS 91, Namur (12-16 Avril 93) 217-220.

[13] E. Papirer, H. Balard, Y. Rahmani, L. Facchini, H. Hommel, Chromatographia 23 (1987) 639-647.

[14] C. Charnay, Doctorate thesis, University of de Montpellier II, 1999. 